\title{
Nailfold capillaroscopy by smartphone-dermatoscope for connective tissue disease diagnosis in interstitial lung disease: a prospective observational study
}

\author{
Adelle S. Jee $\mathbb{1}^{1,2,3}$, Matthew J.S. Parker ${ }^{2,3,4}$, Neil McGill ${ }^{2,4}$, Jane F. Bleasel ${ }^{2,4}$, Susanne Webster ${ }^{1}$, \\ Lauren K. Troy ${ }^{1,2}$ and Tamera J. Corte $\mathrm{e}^{1,2,3}$
}

${ }^{1}$ Dept of Respiratory, Royal Prince Alfred Hospital, Sydney, NSW, Australia. ${ }^{2}$ Sydney Medical School, The University of Sydney, Sydney, NSW, Australia. ${ }^{3}$ National Health and Medical Research Council Centre of Research Excellence in Pulmonary Fibrosis, Sydney, Australia. ${ }^{4}$ Dept of Rheumatology, Royal Prince Alfred Hospital, Sydney, NSW, Australia.

Corresponding author: Adelle S. Jee (adelle.jee@health.nsw.gov.au)

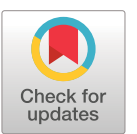

Copyright OThe authors 2021

This version is distributed under the terms of the Creative Commons Attribution NonCommercial Licence 4.0. For commercial reproduction rights and permissions contact permissions@ersnet.org

This article has supplementary material available from openres.ersjournals.com

Received: 23 June 2021 Accepted: 2 Aug 2021

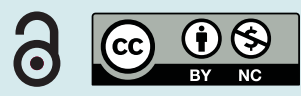

Shareable abstract (@ERSpublications)

Nailfold capillaroscopy by smartphone-dermatoscope is a safe, feasible tool that may improve the identification of connective tissue disease associated with interstitial lung disease beyond routine clinical assessment https://bit.ly/3iLjxUy

Cite this article as: Jee AS, Parker MJS, McGill N, et al. Nailfold capillaroscopy by smartphonedermatoscope for connective tissue disease diagnosis in interstitial lung disease: a prospective observational study. ERJ Open Res 2021; 7: 00416-2021 [DOI: 10.1183/23120541.00416-2021].

\section{Abstract}

Nailfold capillaroscopy (NFC) is a non-invasive tool validated for systemic sclerosis diagnosis. The role and interpretation of NFC in interstitial lung disease (ILD) patients for the diagnosis of connective tissue disease associated ILD (CTD-ILD) remains undefined.

In a prospective study, quantitative and qualitative NFC by smartphone-dermatoscope (3M DermliteDL4TM attached to iPhone-6plusTM) was performed in 96 patients with well-defined CTD-ILD ( $\mathrm{n}=27$ ) and non-CTD ILD ( $n=69$; idiopathic interstitial pneumonia $n=42$, interstitial pneumonia with autoimmune features $\mathrm{n}=27$ ) by ILD-multidisciplinary meeting. NFC scoring was performed by two independent, blinded specialist rheumatologists. Comprehensive baseline clinical, serological, physiological and radiological data were included. Multivariable models for CTD diagnosis in ILD, comprising nailfold characteristics at empirical thresholds determined by receiver operating characteristic curve analysis and clinical variables, were explored.

In 94 patients with complete NFC data (total 687 images, median eight images per patient from eight digits), low capillary density ( $<6$ capillaries/millimetre), increased giant capillaries $(\geqslant 3)$, avascular areas $(\geqslant 2)$ and microhaemorrhages all strongly enhanced the discrimination of CTD-ILD from non-CTD ILD (OR 5.00-7.47) independent of clinical covariates. In multivariable analysis, low capillary density and microhaemorrhages were independent predictors of CTD in ILD additional to the risk conferred by serology and radiology. Microhaemorrhages were also a strong predictor of CTD (adjusted OR 13.45, $\mathrm{p}=0.006$ ) independent of clinical manifestations. All pre-specified qualitative NFC classification schemes identified CTD-ILD (OR range 3.27-8.47).

NFC performed by smartphone-dermatoscope is an accessible, clinically feasible tool that may improve the identification of CTD further to routine clinical assessment of the ILD patient.

\section{Introduction}

Interstitial lung disease (ILD) can be associated with all connective tissue diseases (CTDs), and when present is a major cause of morbidity and mortality. Accordingly, international guidelines recommend routine assessment for CTD in all ILD patients [1]. CTD-ILD most often manifests in the context of an established CTD, but the ILD may be the first or only presentation of CTD and can be difficult to distinguish from idiopathic interstitial pneumonia (IIP) [2].

Nailfold capillaroscopy (NFC) is a noninvasive tool validated for the diagnosis of systemic sclerosis (SSc) and distinguishing primary from secondary Raynaud's phenomenon, with potential utility in a broader 
array of CTDs [3, 4]. Quantitative and qualitative nailfold features have been associated with pulmonary involvement in CTD [5-7]. Thus, NFC has been proposed as a screening tool for CTD in ILD patients. However, a paucity of studies in non-rheumatological cohorts leaves the role and interpretation of NFC in ILD patients undefined. Furthermore, gold-standard capillaroscopic techniques, widefield stereomicroscopy and videocapillaroscopy, are expensive with limited access in many clinical settings. Thus, there is increasing interest in lower-cost, portable devices that can be easily applied in clinical practice, including the "smartphone-dermatoscope" [8].

Our primary objective was to describe quantitative and qualitative nailfold characteristics by smartphone-dermatoscope in well-defined cohorts of CTD-ILD and non-CTD-ILD, (comprised of IIP and interstitial pneumonia with autoimmune features (IPAF)), at a tertiary ILD referral centre. Secondary objectives included evaluation of the association of nailfold characteristics with CTD diagnosis and clinical variables in ILD. Empirical thresholds of nailfold characteristics to identify CTD in ILD were calculated. The association of nailfold characteristics with CTD in ILD together with clinical, serological and radiological variables were explored.

\section{Methods}

Study design and participants

Consecutive patients attending a specialist ILD clinic were prospectively screened for inclusion (August 18, 2016 to January 24, 2018). Eligible patients were aged $\geqslant 18$ years, with a consensus diagnosis of CTD-ILD or IIP by ILD multidisciplinary meeting (ILD-MDM), and able to give informed consent. CTD diagnoses were defined by international criteria for SSc [3], rheumatoid arthritis [9], Sjögren’s syndrome [10], mixed connective tissue disease [11], idiopathic inflammatory myositis (IIM) [12] and systemic lupus erythematosus [13]. IIPs were defined by American Thoracic Society/European Respiratory Society classification criteria [1, 14]. Of eligible participants, patients meeting IPAF criteria were separately identified [15]. CTD-ILD and IPAF classifications were confirmed by specialist rheumatologist assessment. Patients unable to provide consent or without CTD-ILD or IIP by ILD-MDM consensus were excluded. Diagnosis at follow-up was censored on June 15, 2020. Ethical approval was granted by the Sydney Local Health District human ethics committee (protocol number X16-0111 HREC/16/RPAH/137).

\section{Definitions}

To delineate a well-defined CTD-ILD cohort for analysis, “CTD-ILD” included only participants fulfilling CTD classification criteria as specified. "Non-CTD-ILD” included IIP and IPAF participants. Disease duration was defined as time from onset of symptoms to first clinic presentation.

Considering IPAF criteria, participants with positive anti-tRNA synthetase autoantibodies, amyopathic disease, and no other features diagnostic of anti-synthetase syndrome were classified as IPAF. Anti-Ro52, anti-Mi2 and anti-SRP were considered as meeting serological IPAF criteria. "Unexplained vasculopathy" was defined as pulmonary hypertension $(\mathrm{PH})$ on echocardiogram (systolic pulmonary arterial pressure (PAP) $>35 \mathrm{mmHg}$ above right atrial pressure) [16] or right heart catheterisation (RHC; mean PAP $\geqslant 25 \mathrm{mmHg}$ ) with forced vital capacity (FVC) $>70 \%$ predicted. "Unexplained airway disease" was defined as forced expiratory volume in $1 \mathrm{~s}\left(\mathrm{FEV}_{1}\right) / \mathrm{FVC}$ ratio $<70 \%$ with no history of asthma, chronic obstructive airways disease or smoking.

\section{Data collection}

Standardised clinical assessment, extended autoantibody testing (antinuclear antibodies (ANAs), extractable nuclear antibodies (ENAs) (Ro-60/SS-A (Sjogren's syndrome-related antigen-A), Ro-52, La/SS-B (Sjogren's syndrome-related antigen-B), ribonuclear protein (RNP), Scl-70, Smith, centromere, polymerase delta auxiliary protein (PCNA), ribosomal-P), myositis antigens (Mi-2, Ku, PM-Scl100, PM-Scl75, Jo-1, SRP, PL-7, PL-12, EJ, OJ; Euroimmun Myositis Profile 3), dsDNA, RF, CCP, ANCA, MPO and PR-3; non-abbreviated labels provided in supplementary table S1), lung function testing and NFC were performed at baseline. ANA titre $\geqslant 1: 320$ or any titre if a nucleolar or centromere pattern were considered positive [15].

Pulmonary physiological indices included $\mathrm{FEV}_{1}, \mathrm{FVC}$, diffusing capacity for carbon monoxide $\left(D_{\mathrm{LCO}}\right)$, peripheral oxyhaemoglobin saturation $\left(S_{\mathrm{pO}_{2}}\right)$, 6-min walk test (6MWT) distance and $S_{\mathrm{pO}_{2}}$ nadir. Composite physiological index (CPI) and ILD gender-age-physiology (ILD-GAP) stage were calculated [17, 18].

Radiological pattern on high-resolution computed tomography and histopathology results reported by specialist radiologist and pathologist at ILD-MDM were obtained from medical records. The presence of 
PH was determined from echocardiogram and/or RHC results as available. Treatment remained as per the attending physician.

\section{Nailfold capillaroscopy}

A single clinician (AJ) performed NFC with a smartphone-dermatoscope (3M Dermlite DL4 ${ }^{\mathrm{TM}}$ (3Gen Inc., San Juan Capistrano, CA, USA) attached to iPhone 6plus ${ }^{\mathrm{TM}}$, figure 1) on eight digits, excluding thumbs, at 20-times optical magnification after a period of acclimatisation at room temperature $\left(20-24^{\circ} \mathrm{C}\right)$. A photograph of a measurement ruler taken at the same magnification was included with the images for assessment. The centre $3 \mathrm{~mm}$ of each image was scored by two independent, blinded specialist rheumatologists (NM, MP) and recorded on a prespecified digital form (supplementary figure S2). Discordant results were discussed by the two scorers to reach consensus. Nailfold characteristics recorded for each image included: (1) capillary density (number of capillary loops in the most distal capillary row per millimetre); total number of (2) microhaemorrhages (haemosiderin deposits in the cuticle, not related to trauma); (3) giant capillaries (capillaries more than four times normal capillary size); (4) avascular areas (distinct areas $>0.5 \mathrm{~mm}$ in the distal capillary row with no capillaries visible); and (5) abnormal capillary shapes (enlarged ( $<4$ times normal), tortuous (capillary width $>2$ times apex width without capillary limb enlargement) or abrogated/“bushy” capillary loops). Figure 1 depicts capillaroscopic images and techniques. Abnormal capillary shapes (ACS) were recorded by semiquantitative score reflecting percentage of capillaries affected: $0=<10 \%$ of capillaries demonstrating ACS; $1=10-50 \%$; $2=>50 \%$. Qualitative scoring was performed using prespecified Ingegnoli, Cutolo and Maricq criteria [19-21]. Patients meeting 2020 European League Against Rheumatism (EULAR) Study Group on Microcirculation in Rheumatic Disease (SG-MC) consensus for "scleroderma", published after initial qualitative data scoring, were identified post hoc using quantitative data [22].

Study data were collected and managed using REDCap hosted at The University of Sydney [23].
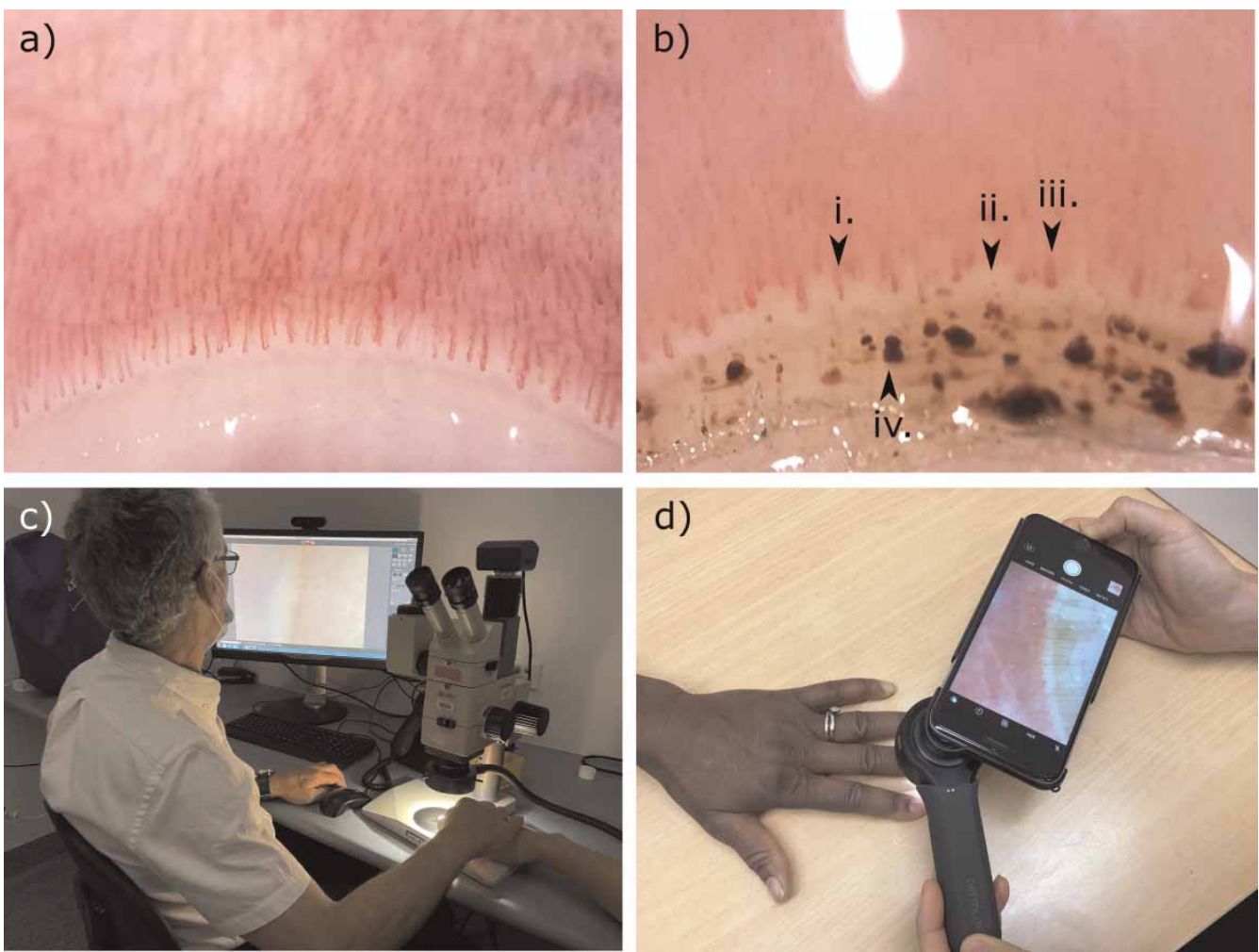

FIGURE 1 Nailfold patterns, characteristics and technique. a) Normal nailfold capillaroscopy pattern;

b) "scleroderma" pattern with (i) abnormal capillary shape, (ii) avascular area, (iii) giant capillary and (iv) microhaemorrhages; c) capillaroscopy by widefield stereomicroscopy; d) nailfold capillaroscopy by "smartphone-dermatoscope". 


\section{Statistical analysis}

Comparisons between ILD groups were performed using two-tailed t-test or Fisher's exact test as appropriate. Continuous quantitative nailfold characteristics (mean capillary density, number of giant capillaries, avascular areas, microhaemorrhages, ACS) were evaluated for an empirical threshold using receiver operating characteristic (ROC) curve analysis and Youden index to maximise the sum of sensitivity and specificity. Threshold values were rounded to the closest whole number for pragmatic assessment. Univariable and multivariable logistic regression (adjusted for age, sex, smoking, FVC \% predicted (FVC\%), $\mathrm{PH}$ and treatment) were used to evaluate associations between nailfold thresholds and clinical variables for the identification of CTD in ILD.

Variables with an area under the ROC curve (AUC) $>0.6$ and p-value $<0.10$ in univariable analyses were retained in exploratory multivariable analyses. Collinear variables were identified by pairwise correlation $(r>0.5)$ and removed from further analysis. Independent predictors of CTD in ILD were identified using backward stepwise selection and the Akaike Information Criterion, further adjusted for age, sex, smoking, FVC\%, PH and treatment. The association of nailfold characteristics with CTD diagnosis in ILD in the absence of clinically overt CTD manifestations was explored.

Inter-rater reliability of mean capillary density, total number of giant capillaries, microhaemorrhages, avascular areas and ACS was assessed by intraclass correlation coefficients.

Continuous variables are reported as mean \pm SD and categorical variables as absolute number (relative frequency). Missing data were not estimated and removed from the denominator when calculating relative frequencies. p-values $<0.05$ were considered statistically significant. Statistical analysis was performed using Stata statistical software (v14.0, StataCorp, College Station, TX, USA) and GraphPad Prism (v8.3.0, GraphPad, San Diego, CA, USA).

Results

96 patients met inclusion criteria, including 27 (28.1\%) with definite CTD-ILD and 69 (71.9\%) with non-CTD-ILD (IIP n=42 (43.8\%), IPAF n=27 (28.1\%)). Specific diagnoses are shown in figure 2.

\section{Baseline characteristics}

Baseline characteristics are shown in table 1 . Mean age was $66.4 \pm 10.3$ years, $44.8 \%(n=43)$ female, $57.3 \%$ ( $n=55)$ ever-smokers, mean FVC $73.9 \%$ and $D_{\text {LCO }} 54.8 \%$ predicted. PH was present in 12 (14.5\%) of 83 patients with available echocardiogram or RHC data and did not differ between ILD subgroups.

CTD-ILD patients were younger, more female predominant and less likely to be smokers compared with non-CTD-ILD patients. Disease duration was longer in CTD-ILD, with no difference in respiratory symptom duration. CTD-ILD patients overall had more favourable physiology (table 1).

Clinical, serological and radiological characteristics are detailed in supplementary table S3 (for ILD subgroup comparison see supplementary table S4). At baseline, treatment for ILD was more common in CTD-ILD compared with non-CTD-ILD patients ( $p=0.017$; supplementary table S5).

\section{Nailfold capillaroscopy characteristics at baseline}

Baseline NFC was available in 94 patients (total 687 images, median eight images per patient (interquartile range 7-8); two patients excluded with insufficient image quality).

CTD-ILD patients demonstrated lower mean capillary density; higher prevalence of giant capillaries, avascular areas and microhaemorrhages; and a greater number of giant capillary and avascular areas compared with non-CTD-ILD patients (table 2). Nailfold characteristics did not differ between IIP and IPAF patients.

There were no correlations between nailfold characteristics and age, symptom duration or physiology $\left(S_{\mathrm{pO}_{2}}\right.$, FVC\%, $D_{\text {LCO }} \%$, CPI).

Inter-rater reliability of low capillary density was excellent (intraclass correlation coefficient=0.90), microhaemorrhages good (ICC $=0.81$ ) and remaining nailfold characteristics moderate (supplementary table S6) [24]. 
Screened for inclusion

ILD-MDM diagnosis for CTD-ILD or IIP $n=100$

Meets diagnostic criteria for definite CTD

\begin{tabular}{|l|}
\multicolumn{1}{|c|}{} \\
\multicolumn{1}{|c|}{ CTD-ILD n=27 } \\
$9(33.3 \%)$ SSC \\
$5(18.5 \%)$ IIM \\
$5(18.5 \%)$ MCTD \\
$3(11.1 \%)$ RA \\
$3(11.1 \%)$ SLE \\
$2(7.4 \%)$ PSA \\
\hline
\end{tabular}

Autoimmune features without characterisable CTD

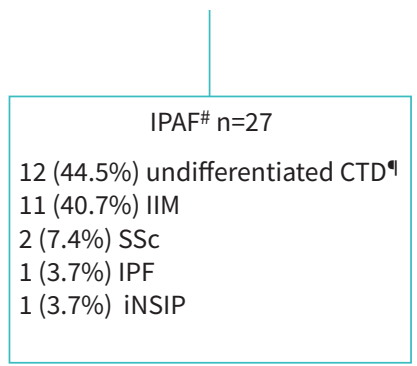

Excluded $\mathrm{n}=4$ Withdrew: $\mathrm{n}=1$

Change in diagnosis after screening: unclassifiable ILD $\mathrm{n}=2$

bronchiectasis without ILD $n=1$

FIGURE 2 Study flowchart and ILD diagnoses. ILD-MDM: interstitial lung disease multidisciplinary meeting; CTD-ILD: connective tissue disease associated ILD; IIP: idiopathic interstitial pneumonia; IPAF: interstitial pneumonia with autoimmune features; SSc: systemic sclerosis; IIM: idiopathic inflammatory myositis; MCTD: mixed connective tissue disease; RA: rheumatoid arthritis; SLE: systemic lupus erythematosus; PsA: psoriatic arthritis; IPF: idiopathic pulmonary fibrosis; iNSIP: idiopathic nonspecific interstitial pneumonia; iOP: idiopathic organising pneumonia. ": IPAF - "working diagnosis" based on clinical, serological and radiological features as per ILD-MDM discussion. ": Undifferentiated CTD = features of autoimmune disease without an identifiable provisional phenotype.

\section{Nailfold capillaroscopy for CTD diagnosis in ILD}

Empirical thresholds of nailfold characteristics with the greatest sensitivity and specificity to identify CTD in ILD were $<6$ capillaries $/ \mathrm{mm}, \geqslant 3$ giant capillaries, $\geqslant 2$ avascular areas and $\geqslant 1$ microhaemorrhage (ROC curve analysis shown in supplementary figure S7). ACS were present in all CTD-ILD patients and omitted from further analysis.

In univariable analysis, all nailfold characteristics at empirical thresholds identified CTD in ILD (unadjusted OR 5.00-7.47), maintained with multivariable adjustment (table 3). The presence of clinical CTD manifestations (any of inflammatory arthritis, Raynaud's phenomenon, digital oedema, palmar telangiectasia, digital tip ulceration, mechanic's hands, Gottron's papules/sign, sclerodactyly) had the highest discriminative performance for CTD-ILD diagnosis relative to non-CTD-ILD (AUC 0.86).

Giant capillaries and avascular areas demonstrated collinearity with capillary density and were excluded from further analysis. Variables that remained for inclusion in exploratory multivariable analyses included capillary density, microhaemorrhages, age, sex, smoking, $D_{\mathrm{LCO}} \%$, CTD manifestations, ANAs, ENAs and radiological nonspecific interstitial pneumonia (NSIP), organising pneumonia or NSIP/organising pneumonia (multivariable regression of all retained variables shown in supplementary table S8). Respective to all included components, microhaemorrhages (adjusted OR 13.45, 95\% CI 2.14-84.32, p=0.006) and CTD manifestations were identified as strong independent predictors of CTD diagnosis in ILD, including after adjustment for age, sex, smoking, FVC\%, PH and treatment (table 4). In the absence of CTD manifestations, microhaemorrhages, low capillary density and positive ENA remained as independent predictors of CTD-ILD from non-CTD-ILD (table 4; included components see supplementary table S8). 


\begin{tabular}{|c|c|c|c|c|c|}
\hline & \multirow[t]{2}{*}{ TOTAL } & \multirow[t]{2}{*}{ CTD-ILD } & \multicolumn{2}{|c|}{ Non-CTD-ILD } & \multirow{2}{*}{$\begin{array}{c}\text { CTD-ILD versus } \\
\text { non-CTD-ILD } \\
\text { p-value }\end{array}$} \\
\hline & & & IPAF & IIP & \\
\hline Subjects n & 96 & 27 & 27 & 42 & \\
\hline Age years & $66.4 \pm 10.3$ & $61.5 \pm 8.9$ & $64.2 \pm 11.1$ & $71.0 \pm 8.8$ & 0.003 \\
\hline Female n (\%) & $43(44.8)$ & $20(74.1)$ & $15(55.6)$ & $8(19.0)$ & $<0.001$ \\
\hline Smoking ever $\mathrm{n}(\%)$ & $55(57.3)$ & $10(37.0)$ & $16(59.3)$ & $29(69.1)$ & 0.021 \\
\hline Caucasian n (\%) & $79(82.3)$ & $19(70.4)$ & $23(85.2)$ & $37(88.1)$ & 0.075 \\
\hline Disease duration years & $7.4 \pm 7.7$ & $11.3 \pm 10.6$ & $4.3 \pm 3.0$ & $6.9 \pm 6.5$ & 0.002 \\
\hline Respiratory symptoms years & $5.7 \pm 5.4$ & $5.6 \pm 4.8$ & $4.3 \pm 3.0$ & $6.6 \pm 6.7$ & 0.292 \\
\hline \multicolumn{6}{|l|}{ Physiology } \\
\hline$S_{\mathrm{pO}_{2}} \%$ & $96.6 \pm 2.5$ & $97.9 \pm 2.4$ & $96.7 \pm 2.3$ & $95.8 \pm 2.4$ & 0.003 \\
\hline FVC \% & $73.9 \pm 17.8$ & $77.0 \pm 17.7$ & $72.3 \pm 18.8$ & $72.8 \pm 17.5$ & 0.281 \\
\hline$D_{\mathrm{LCO}} \%$ & $54.8 \pm 16.6$ & $60.7 \pm 19.8$ & $54.8 \pm 16.2$ & $50.9 \pm 13.6$ & 0.028 \\
\hline 6 MWT distance metres & $446.5 \pm 126.1^{+}$ & $463.0 \pm 88.6^{4}$ & $421.5 \pm 138.6^{f}$ & $453.1 \pm 137.3^{\# \#}$ & 0.479 \\
\hline $6 \mathrm{MWT} \mathrm{S}_{\mathrm{pO}_{2}}$ nadir $\%$ & $90.0 \pm 7.1^{+}$ & $94.3 \pm 3.6^{4}$ & $90.0 \pm 7.9^{f}$ & $87.6 \pm 7.2^{\# \#}$ & 0.001 \\
\hline \multicolumn{6}{|l|}{ Composite indices } \\
\hline CPI & $43.1 \pm 12.8$ & $38.9 \pm 14.2$ & $46.2 \pm 10.4$ & $46.2 \pm 10.4$ & 0.044 \\
\hline ILD-GAP score & $1.9 \pm 2.2$ & $0 \pm 1.6$ & $1.2 \pm 1.5$ & $2.5 \pm 1.5$ & $<0.001$ \\
\hline
\end{tabular}

Data represented as mean \pm SD unless otherwise stated. CTD-ILD: connective tissue disease associated ILD; IPAF: interstitial pneumonia with autoimmune features; IIP: idiopathic interstitial pneumonia; $\mathrm{S}_{\mathrm{pO}_{2}}$ : peripheral oxygen saturation; FVC \%: percentage predicted forced vital capacity; $D_{\mathrm{LcO}} \%$ : percentage predicted diffusing capacity for carbon monoxide; 6MWT: 6-min walk test; CPI: composite physiological index; ILD-GAP ILD: gender-agephysiology index. ${ }^{\#}$ : $p$-value for CTD-ILD versus non-CTD-ILD; ${ }^{9}: n=22 ;{ }^{+}: n=82 ;{ }^{\S}: n=60 ;{ }^{f}: n=24 ;{ }^{\# \#}: n=36$.

\section{Qualitative nailfold capillaroscopy analysis}

A "scleroderma" or "active/late" pattern by all prespecified qualitative classification criteria identified CTD-ILD (table 5). Frequency of qualitative patterns by ILD group are shown in supplementary table S9. Four non-CTD-ILD patients (IIP n=2, IPAF n=2) demonstrated a "scleroderma" or "active/late" pattern across all criteria (clinical details shown in supplementary table S10).

\section{Follow-up}

Mean follow-up time was 2.5 years (range 72 days to 3.8 years). No non-CTD-ILD patients with abnormal NFC at baseline developed a diagnostic CTD during the study period.

TABLE 2 Nailfold capillaroscopy characteristics by interstitial lung disease (ILD) group

\begin{tabular}{|c|c|c|c|c|c|}
\hline & \multirow[t]{2}{*}{ Total } & \multirow[t]{2}{*}{ CTD-ILD } & \multicolumn{2}{|c|}{ Non-CTD-ILD } & \multirow[t]{2}{*}{ CTD-ILD versus non-CTD-ILD p-value } \\
\hline & & & IPAF & IIP & \\
\hline Subjects $\mathrm{n}$ & 94 & 26 & 27 & 41 & \\
\hline \multicolumn{6}{|l|}{ Density } \\
\hline Number per mm & $6.7 \pm 1.4$ & $5.6 \pm 1.6$ & $6.8 \pm 1.1$ & $7.3 \pm 0.9$ & $<0.001$ \\
\hline \multicolumn{6}{|l|}{ Giant capillaries } \\
\hline Present n (\%) & $38(40.4)$ & $16(61.5)$ & $12(44)$ & $10(24.4)$ & 0.018 \\
\hline Number per patient & $2.9 \pm 6.3$ & $7.0 \pm 1.1$ & $2.8 \pm 6.1$ & $0.5 \pm 8.9$ & $<0.001$ \\
\hline \multicolumn{6}{|l|}{ Avascular areas } \\
\hline Present n (\%) & $36(38.3)$ & $15(57.7)$ & $12(44.4)$ & $9(22.0)$ & 0.031 \\
\hline Number per patient & $1.3 \pm 2.2$ & $2.7 \pm 3.2$ & $1.2 \pm 1.6$ & $0.5 \pm 1.1$ & $<0.001$ \\
\hline \multicolumn{6}{|l|}{ Microhaemorrhages } \\
\hline Present n (\%) & $72(76.6)$ & $24(92.3)$ & $18(66.7)$ & $30(73.2)$ & 0.030 \\
\hline Number per patient & $5.8 \pm 12.1$ & $4.9 \pm 6.7$ & $8.2 \pm 18.0$ & $4.9 \pm 9.9$ & 0.640 \\
\hline \multicolumn{6}{|c|}{ Abnormal capillary shapes } \\
\hline Present $\mathrm{n}(\%)$ & $90(95.7)$ & $26(100)$ & $24(88.9)$ & $40(97.6)$ & 0.573 \\
\hline Mode score & $0.7 \pm 0.8$ & $1.2 \pm 0.8$ & $0.7 \pm 0.8$ & $0.4 \pm 0.6$ & $<0.001$ \\
\hline
\end{tabular}

Data represented as mean \pm SD unless otherwise stated. CTD-ILD: connective tissue disease associated ILD; IPAF: interstitial pneumonia with autoimmune features; IIP: idiopathic interstitial pneumonia. 


\begin{tabular}{|c|c|c|c|c|}
\hline & Unadjusted OR & $95 \% \mathrm{Cl}$ & p-value & AUC \\
\hline \multicolumn{5}{|l|}{ Nailfold characteristics } \\
\hline Density $<6 / \mathrm{mm}$ & 7.47 & $2.73-20.43$ & $<0.001^{\#}$ & 0.72 \\
\hline Giant $\geqslant 3$ total & 6.77 & $2.43-18.81$ & $<0.001^{\#}$ & 0.70 \\
\hline Avascular $\geqslant 2$ total & 5.44 & $2.02-14.68$ & $0.001^{\#}$ & 0.68 \\
\hline Microhaemorrhages $\geqslant 1$ total & 5.00 & $1.08-23.18$ & $0.040^{\#}$ & 0.61 \\
\hline \multicolumn{5}{|l|}{ Baseline characteristics } \\
\hline Age & 0.94 & $0.89-0.98$ & $0.005^{\#}$ & 0.70 \\
\hline Male & 0.18 & $0.06-0.47$ & 0.001 & 0.70 \\
\hline Smoking & 0.31 & $0.12-0.79$ & 0.014 & 0.64 \\
\hline FVC $\%$ predicted & 1.01 & $0.99-1.04$ & 0.279 & 0.59 \\
\hline$D_{\mathrm{Lco}} \%$ predicted & 1.03 & $1.00-1.06$ & 0.032 & 0.64 \\
\hline \multicolumn{5}{|l|}{ Clinical CTD manifestations } \\
\hline Any CTD manifestation & 42.18 & $10.80-164.74$ & $<0.001^{\#}$ & 0.86 \\
\hline \multicolumn{5}{|l|}{ Serology and radiology } \\
\hline ANA $\geqslant 1: 320$ & 3.80 & $1.42-10.14$ & 0.008 & 0.64 \\
\hline Positive ENA & 5.38 & $2.04-14.20$ & $0.001^{\#}$ & 0.68 \\
\hline Any myositis autoantibody ${ }^{+}$ & 0.33 & $0.10-1.05$ & 0.061 & 0.60 \\
\hline Radiological NSIP, OP or NSIP/OP & 7.48 & $2.33-23.93$ & $0.001^{\#}$ & 0.71 \\
\hline \multicolumn{5}{|c|}{$\begin{array}{l}\text { Odds ratios (OR) are shown for the bivariate relationship of each variable with CTD-ILD diagnosis. FVC \%: } \\
\text { percentage predicted forced vital capacity; } D_{\text {LCO }} \% \text { : percentage predicted diffusing capacity for carbon } \\
\text { monoxide; ANA: antinuclear antibodies; ENA: extractable nuclear antibodies; NSIP: nonspecific interstitial } \\
\text { pneumonia pattern on radiology; OP: organising pneumonia pattern on radiology; ": remains significant } \\
\text { adjusted for age, sex, smoking, FVC\%, treatment and pulmonary hypertension; ‘: including any of inflammatory } \\
\text { arthritis, Raynaud's phenomenon, digital oedema, palmar telangiectasia, digital tip ulceration, mechanic's } \\
\text { hands, Gottron's papules/sign, sclerodactyly; }{ }^{+}: \text {patients with positive myositis autoantibody and no clinical } \\
\text { features of myositis classified as IPAF (non-CTD-ILD). }\end{array}$} \\
\hline
\end{tabular}

\section{Discussion}

We describe NFC by smartphone-dermatoscope in 94 patients with well-defined ILD and demonstrate its potential to identify CTD in ILD patients further to multidisciplinary assessment at empirical thresholds. NFC has been proposed as a noninvasive tool to screen for CTD in ILD patients, but a paucity of studies in dedicated ILD cohorts has limited its use and interpretation in clinical practice. We have demonstrated the feasibility and utility of NFC by smartphone-dermatoscope in the ILD clinical setting, and its potential to improve the identification of CTD further to standard clinical assessment.

To our knowledge, our study is the first to evaluate quantitative NFC in well-characterised ILD populations and its combination with clinical variables including empirical thresholds. Low capillary density, increased giant capillaries, avascular areas and microhaemorrhages all strongly enhanced the discrimination of CTD-ILD from non-CTD-ILD, independent of baseline age, sex, smoking history, FVC\%, prevalent $\mathrm{PH}$

TABLE 4 Independent predictors for connective tissue disease (CTD) diagnosis in interstitial lung disease (ILD) identified by exploratory multivariable regression

\begin{tabular}{lcccc} 
& Predictors $^{\#}$ & Adjusted OR & 95\% Cl & p-value \\
\hline A) Including CTD manifestations & Any CTD manifestation & 62.84 & $13.93-283.40$ & $<0.001$ \\
& Microhaemorrhages & 13.45 & $2.14-84.32$ & 0.006 \\
B) Excluding CTD manifestations & Positive ENA & 11.59 & $1.80-74.55$ & 0.010 \\
& Microhaemorrhages & 22.54 & $1.89-269.05$ & 0.014 \\
& Low capillary density & 5.66 & $1.32-24.20$ & 0.019 \\
\hline
\end{tabular}

A) Independent predictors for CTD diagnosis in ILD respective to retained nailfold characteristics, clinical, serological and radiological variables. B) Independent predictors for CTD diagnosis in ILD excluding CTD manifestations, respective to remaining nailfold characteristics, serological and radiological variables. ENA: extractable nuclear antibodies. \#: all retained variables in initial multivariable regression shown in supplementary table S8; ฯ": adjusted for age, sex, smoking, FVC\%, treatment and pulmonary hypertension. 


\begin{tabular}{|c|c|c|c|c|}
\hline Proposed criteria & Pattern & Unadjusted $\mathrm{OR}^{\#}$ & $95 \% \mathrm{Cl}$ & $p$-value \\
\hline \multirow[t]{4}{*}{ INGEGNOLI [19] } & Normal & 1 & & \\
\hline & Minor & 2.15 & $0.58-7.98$ & 0.254 \\
\hline & Major & 3.02 & $0.67-13.63$ & 0.015 \\
\hline & Scleroderma & 15.30 & $3.37-68.99$ & $<0.001^{\natural}$ \\
\hline \multirow[t]{3}{*}{ MARICQ [21] } & Normal & 1 & & \\
\hline & Nonspecific & 2.05 & $0.58-7.22$ & 0.262 \\
\hline & Scleroderma & 9.39 & $2.80-31.45$ & $<0.001^{\circ}$ \\
\hline \multirow[t]{4}{*}{ Cutolo [20] } & Normal & 1 & & \\
\hline & Early & 2.01 & $0.57-7.04$ & 0.276 \\
\hline & Active & 6.09 & $1.66-22.42$ & $0.007^{9}$ \\
\hline & Late & 15.67 & $1.46-168.07$ & $0.023^{4}$ \\
\hline EULAR SG-MC [22] & Scleroderma & 3.91 & $1.47-10.42$ & $0.006^{4}$ \\
\hline
\end{tabular}

and treatment. CTD manifestations were unequivocally the strongest predictor of CTD, underlining that careful clinical assessment remains at the core of accurate diagnosis. Nevertheless, confirming a diagnosis of CTD in ILD patients can remain elusive despite comprehensive multidisciplinary assessment, owing to disease heterogeneity, and in particular, occult or clinically amyopathic CTD. Surprisingly, microhaemorrhages remained a strong predictor for CTD in our ILD cohort after accounting for all other clinical, serological and radiological findings, including CTD manifestations, despite a weaker association in univariable analysis. Encouragingly, in the absence of CTD manifestations, low capillary density and microhaemorrhages were independent predictors of CTD-ILD relative to non-CTD-ILD, signalling the potential utility of NFC to identify occult CTD in ILD populations. Lower capillary density by videocapillaroscopy, but not microhaemorrhages, has been associated with SSc-ILD in prior studies, acknowledging different study populations and capillaroscopy techniques [5-7]. Whilst the optimal combination of nailfold characteristics and clinical variables requires validation, taken together, our data demonstrate the potential of NFC as an additional tool to aid CTD identification together with ILD assessment following international guidelines.

Qualitative assessment further supported the utility of NFC to identify CTD in ILD, regardless of putative classification criteria (OR range 3.27-8.47). However, derived thresholds of nailfold characteristics that optimally identified CTD in our ILD cohort differed from those recommended for SSc-spectrum diseases [22]. We also observed a high prevalence of major capillaroscopic abnormalities (giant capillaries, avascular areas) in patients without a definable CTD-ILD. These data raise the important question of how to define "abnormal” NFC in ILD populations. Capillaroscopy studies in non-CTD-ILD populations are limited, but prior IIP cohorts have reported major nailfold abnormalities in 5.7\% to 46.7\% [25-27]. No non-CTD-ILD patients with nailfold abnormalities developed a definable CTD during our study, recognising that diagnostic CTD features can develop many years after ILD onset [2]. Long-term studies of heterogeneous ILD cohorts are required to characterise the pathogenic mechanisms, clinical implications and outcomes of microvascular changes in non-CTD-ILD patients. We suggest caution extrapolating prespecified nailfold criteria established in rheumatology populations to broader ILD cohorts until further validation.

We have clearly demonstrated the feasibility of NFC by smartphone-dermatoscope in a real-world clinical setting, which may serve as an accessible, lower-cost screening test for CTD in ILD patients. This may guide the need for formal NFC or rheumatologist referral, particularly when there is concern for occult or non-diagnostic CTD features despite standard assessment. Nailfold characteristics retained in our multivariable models, particularly capillary density, have demonstrated ease of measurement and good reproducibility and reliability in our and prior studies [28-30]. The smartphone-dermatoscope is unlikely to replace gold-standard techniques but may strike a pragmatic balance between applicability and performance, with comparable inter- and intra-rater reliability to videocapillaroscopy [8, 31-33]. Its portability and ease-of-use across broad experience levels also improve access to this valuable tool in remote and community settings where specialised services may be limited. Smartphone technology allows 
the assessment of images by remote experts, amplified magnification, comparison of serial imaging and blinding in the research setting. To define the clinical role of NFC in ILD patients, it will be important to understand its impact on diagnostic reasoning, outcomes, and comparison or combination with established and emerging modalities, including USB microscopy and computer-automated scoring systems [34].

Our data support the hypothesis that microvascular dysfunction is a key pathogenic mechanism in pulmonary fibrosis [25, 35]. The lack of correlation between NFC and arterial oxygen saturation $\left(S_{\mathrm{aO}_{2}}\right)$ or $D_{\text {LCO }} \%$ complements the study by CoRRADo et al. [25] demonstrating normal capillaroscopy in COPD patients, suggestive that microvascular changes are not a simple sequela of hypoxia. Integrating these findings with ILD-associated serum biomarkers of vascular remodelling (e.g., vascular endothelial growth factor, endothelin-1, interleukin-8) may provide pathogenic insights on the role of systemic vascular dysfunction in CTD-related and non-CTD ILDs. The theragnostic potential of NFC remains unknown. At present, a lack of standardised treatment guidelines for CTD-ILD and small study numbers limit conclusions on treatment associations. Nintedanib, an antifibrotic able to slow disease progression in SSc-ILD and progressive-fibrosing ILD, has demonstrated vascular remodelling in animal models [36-38]. However, how this relates to its antifibrotic activity in ILD, the impact on microvascular changes represented in NFC, and whether this can be used to guide therapy remains to be determined.

Our study has several limitations. The single-centre design and small numbers necessitate validation in larger, heterogeneous ILD cohorts. Our IIP cohort was predominantly idiopathic pulmonary fibrosis (IPF), reflecting referral patterns to the centre, and further study of non-IPF IIP cohorts is needed. Comparison with formal capillaroscopy was not possible and individual capillary dimensions were only measurable to the nearest $0.2 \mathrm{~mm}$. To minimise misclassification, images were scored by two independent, blinded experts in capillaroscopy. Nevertheless, our results reflect applicability in daily practice, with prior studies demonstrating good comparability between the dermatoscope and videocapillaroscopy, and the potential for operators of varying experience to reliably interpret NFC with brief training [8, 32, 33, 39]. Qualitative assessment by EULAR SG-MC consensus, included to aid comparison with future studies, was calculated post hoc with possible misclassification. Nevertheless, to date, our study remains one of the largest prospective, quantitative NFC studies with ILD diagnosed by ILD-MDM, the gold-standard for ILD diagnosis. All CTD-ILD and IPAF participants were assessed by an expert rheumatologist, allowing delineation of a well-defined CTD-ILD cohort.

In conclusion, NFC is a safe, feasible tool that improves discrimination of CTD further to routine clinical assessment of the ILD patient. The smartphone-dermatoscope holds potential as a pragmatic, reliable capillaroscopic device with increased feasibility across broad experience levels. Longitudinal study in heterogeneous populations will aid more uniform application and interpretation of NFC for CTD diagnosis in ILD patients.

Acknowledgements: The authors would like to acknowledge Caro Badcock (Shimsco Consulting) for her statistical support. REDCap (Research Electronic Data Capture) is a secure, web-based application designed to support data capture for research studies, providing: 1) an intuitive interface for validated data entry; 2) audit trails for tracking data manipulation and export procedures; 3) automated export procedures for seamless data downloads to common statistical packages; and 4) procedures for importing data from external sources. This project was supported by NIH/NCATS Colorado CTSA Grant Number UL1 TR002535. Its contents are the authors' sole responsibility and do not necessarily represent official NIH views.

Provenance: Submitted article, peer reviewed.

Conflict of interest: A.S. Jee has nothing to disclose. M.J.S. Parker has nothing to disclose. N. McGill has nothing to disclose. J.F. Bleasel has nothing to disclose. S. Webster has nothing to disclose. L.K. Troy has nothing to disclose. T.J. Corte reports grants from Boehringer Ingelheim, Roche, Biogen, Galapagos, Actelion and Avalyn; consulting fees from Boehringer Ingelheim and Roche; board participation for Boehringer Ingleheim, Roche, BMS and Ad Alta; and trial steering committees for Roche, BMS and Promedior.

Support statement: A.S. Jee would like to acknowledge funding support from the Lung Foundation Australia David Wilson PhD Scholarship. M.J.S. Parker would like to acknowledge funding support from the Lung Foundation Australia Brian Eaton Memorial PhD Scholarship. The authors would like to acknowledge the support of the National Health and Medical Research Council Centre of Research Excellence in Pulmonary Fibrosis (GNT1116371), supported by foundation partner Boehringer Ingelheim, and programme partners Roche and Galapagos. Funding information for this article has been deposited with the Crossref Funder Registry. 
References

1 Raghu G, Remy-Jardin M, Myers JL, et al. Diagnosis of idiopathic pulmonary fibrosis. an official ATS/ERS/JRS/ ALAT clinical practice guideline. Am J Respir Crit Care Med 2018; 198: e44-e68.

2 Fischer A, du Bois R. Interstitial lung disease in connective tissue disorders. Lancet 2012; 380: 689-698.

3 van den Hoogen F, Khanna D, Fransen J, et al. 2013 classification criteria for systemic sclerosis: an American College of Rheumatology/European League Against Rheumatism collaborative initiative. Ann Rheum Dis 2013; 72: $1747-1755$.

4 Koenig M, Joyal F, Fritzler MJ, et al. Autoantibodies and microvascular damage are independent predictive factors for the progression of Raynaud's phenomenon to systemic sclerosis: a twenty-year prospective study of 586 patients, with validation of proposed criteria for early systemic sclerosis. Arthritis Rheum 2008; 58: 3902-3912.

5 Castellví I, Simeón-Aznar CP, Sarmiento M, et al. Association between nailfold capillaroscopy findings and pulmonary function tests in patients with systemic sclerosis. J Rheumatol 2015; 42: 222-227.

6 Caetano J, Paula FS, Amaral M, et al. Nailfold videocapillaroscopy changes are associated with the presence and severity of systemic sclerosis-related interstitial lung disease. J Clin Rheumatol 2019; 25: e12-e15.

7 Guillen-Del-Castillo A, Simeon-Aznar CP, Callejas-Moraga EL, et al. Quantitative videocapillaroscopy correlates with functional respiratory parameters: a clue for vasculopathy as a pathogenic mechanism for lung injury in systemic sclerosis. Arthritis Res Ther 2018; 20: 281.

8 Parker MJS, Oliffe MT, McGill NW. An evaluation of two novel capillaroscopy techniques in suspected scleroderma-spectrum disorders: a single-centre cross-sectional study. Mod Rheumatol 2018; 28: 676-680.

9 Aletaha D, Neogi T, Silman AJ, et al. 2010 Rheumatoid arthritis classification criteria: an American College of Rheumatology/European League Against Rheumatism collaborative initiative. Arthritis Rheum 2010; 62: 2569-2581.

10 Shiboski CH, Shiboski SC, Seror R, et al. 2016 American College Of Rheumatology/European League Against Rheumatism classification criteria for primary Sjogren's syndrome: a consensus and data-driven methodology involving three international patient cohorts. Arthritis Rheumatol 2016; 69: 35-45.

11 Alarcon-Segovia D, Cardiel MH. Comparison between 3 diagnostic criteria for mixed connective tissue disease. Study of 593 patients. J Rheumatol 1989; 16: 328-334.

12 Hoogendijk JE, Amato AA, Lecky BR, et al. 119th ENMC international workshop: trial design in adult idiopathic inflammatory myopathies, with the exception of inclusion body myositis, 10-12 October 2003, Naarden, The Netherlands. Neuromuscul Disord 2004; 14: 337-345.

13 Petri M, Orbai AM, Alarcon GS, et al. Derivation and validation of the systemic lupus international collaborating clinics classification criteria for systemic lupus erythematosus. Arthritis Rheum 2012; 64: 2677-2686.

14 ATS/ERS. American Thoracic Society/European Respiratory Society international multidisciplinary consensus classification of the idiopathic interstitial pneumonias. Am J Respir Crit Care Med 2002; 165: 277-304.

15 Fischer A, Antoniou KM, Brown KK, et al. An Official European Respiratory Society/American Thoracic Society research statement: interstitial pneumonia with autoimmune features. Eur Respir J 2015; 46: 976-987.

16 Augustine DX, Coates-Bradshaw LD, Willis J, et al. Echocardiographic assessment of pulmonary hypertension: a guideline protocol from the British Society of Echocardiography. Echo Res Pract 2018; 5: G11-G24.

17 Ryerson CJ, Vittinghoff E, Ley B, et al. Predicting survival across chronic interstitial lung disease: the ILD-GAP model. Chest 2014; 145: 723-728.

18 Wells AU, Desai SR, Rubens MB, et al. Idiopathic pulmonary fibrosis: a composite physiologic index derived from disease extent observed by computed tomography. Am J Respir Crit Care Med 2003; 167: 962-969.

19 Ingegnoli F, Zeni S, Gerloni V, et al. Capillaroscopic observations in childhood rheumatic diseases and healthy controls. Clin Exp Rheumatol 2005; 23: 905-911.

20 Cutolo M, Pizzorni C, Tuccio M, et al. Nailfold videocapillaroscopic patterns and serum autoantibodies in systemic sclerosis. Rheumatology (Oxford) 2004; 43: 719-726.

21 Maricq HR, LeRoy EC, D’Angelo WA, et al. Diagnostic potential of in vivo capillary microscopy in scleroderma and related disorders. Arthritis Rheum 1980; 23: 183-189.

22 Smith V, Herrick AL, Ingegnoli F, et al. Standardisation of nailfold capillaroscopy for the assessment of patients with Raynaud's phenomenon and systemic sclerosis. Autoimmun Rev 2020; 19: 102458.

23 Harris PA, Taylor R, Thielke R, et al. Research electronic data capture (REDCap) - a metadata-driven methodology and workflow process for providing translational research informatics support. J Biomed Inform 2009; 42: 377-381.

24 Koo TK, Li MY. A guideline of selecting and reporting intraclass correlation coefficients for reliability research. J Chiropr Med 2016; 15: 155-163.

25 Corrado A, Carpagnano GE, Gaudio A, et al. Nailfold capillaroscopic findings in systemic sclerosis related lung fibrosis and in idiopathic lung fibrosis. Joint Bone Spine 2010; 77: 570-574. 
Sambataro D, Sambataro G, Libra A, et al. Nailfold videocapillaroscopy is a useful tool to recognise definite forms of systemic sclerosis and idiopathic inflammatory myositis in interstitial lung disease patients. Diagnostics (Basel) 2020; 10: 253.

27 Cakmakci Karadogan D, Balkarli A, Onal O, et al. The role of nailfold capillaroscopy in interstitial lung diseases - can it differentiate idiopathic cases from collagen tissue disease associated interstitial lung diseases? Tuberkuloz ve toraks 2015; 63: 22-30.

28 Smith V, Pizzorni C, De Keyser F, et al. Reliability of the qualitative and semiquantitative nailfold videocapillaroscopy assessment in a systemic sclerosis cohort: a two-centre study. Ann Rheum Dis 2010; 69: 1092-1096.

29 Sulli A, Secchi ME, Pizzorni C, et al. Scoring the nailfold microvascular changes during the capillaroscopic analysis in systemic sclerosis patients. Ann Rheum Dis 2008; 67: 885-887.

30 Ingegnoli F, Boracchi P, Gualtierotti R, et al. Prognostic model based on nailfold capillaroscopy for identifying Raynaud's phenomenon patients at high risk for the development of a scleroderma spectrum disorder: PRINCE (Prognostic Index for Nailfold Capillaroscopic Examination). Arthritis Rheum 2008; 58: 2174-2182.

31 Hughes M, Moore T, O'Leary N, et al. A study comparing videocapillaroscopy and dermoscopy in the assessment of nailfold capillaries in patients with systemic sclerosis-spectrum disorders. Rheumatology 2015; 54: $1435-1442$.

32 Dogan S, Akdogan A, Atakan N. Nailfold capillaroscopy in systemic sclerosis: is there any difference between videocapillaroscopy and dermatoscopy? Skin Res Technol 2013; 19: 446-449.

33 Mazzotti NG, Bredemeier M, Brenol CV, et al. Assessment of nailfold capillaroscopy in systemic sclerosis by different optical magnification methods. Clin Exp Dermatol 2014; 39: 135-141.

34 Berks M, Dinsdale G, Marjanovic E, et al. Comparison between low cost USB nailfold capillaroscopy and videocapillaroscopy: a pilot study. Rheumatology 2020; 60: 3862-3867.

35 Bagnato G, Harari S. Cellular interactions in the pathogenesis of interstitial lung diseases. Eur Respir Rev 2015; 24: 102-114.

36 Distler O, Highland KB, Gahlemann M, et al. Nintedanib for systemic sclerosis-associated interstitial lung disease. N Engl J Med 2019; 380: 2518-2528.

37 Flaherty KR, Wells AU, Cottin V, et al. Nintedanib in progressive fibrosing interstitial lung diseases. $N$ Engl J Med 2019; 381: 1718-1727.

38 Wollin L, Distler JHW, Redente EF, et al. Potential of nintedanib in treatment of progressive fibrosing interstitial lung diseases. Eur Respir J 2019; 54: 1900161.

39 Smith V, Vanhaecke A, Herrick AL, et al. Fast track algorithm: how to differentiate a "scleroderma pattern" from a "non-scleroderma pattern". Autoimmun Rev 2019; 18: 102394. 